\title{
Endogenous Information Flows and the Clustering of
}

\author{
Announcements ${ }^{1}$
}

by

\section{Viral V. Acharya, Peter DeMarzo and Ilan Kremer}

\author{
Contact information:
}

\author{
Viral V. Acharya \\ London Business School and CEPR \\ Regent's Park, London - NW1 4SA, UK \\ Tel: +44 (0)207000 8255 \\ E-mail: vacharya@london.edu
}

\author{
Peter DeMarzo \\ Graduate School of Business, Stanford University \\ 518 Memorial Way, Stanford, CA 94305-5015, USA \\ Tel: +1 (650) 736-1082 \\ E-mail: pdemarzo@stanford.edu \\ Ilan Kremer \\ Graduate School of Business, Stanford University \\ 518 Memorial Way, Stanford, CA 94305-5015, USA \\ Tel: +1 (650) 736-0288 \\ E-mail: ikremer@stanford.edu
}

\footnotetext{
${ }^{1}$ A part of this study was undertaken while Viral Acharya was visiting Stanford-GSB. The authors are grateful to Ann Beyer, Martin Dierker (discussant), Mike Fishman, Jennifer Huang (discussant), Jacob Sagi, Jenny Tucker, Florin Vasvari and Laura Veldkamp (discussant) for useful discussions, and to seminar participants at several universities and conferences for useful comments.
} 


\title{
Endogenous Information Flows and the Clustering of Announcements
}

\begin{abstract}
We consider the release of information by a firm when the manager has discretion regarding the timing of its release. While it is well known that firms appear to delay the release of bad news, we examine how external information about the state of the economy (or the industry) affects this decision. We develop a dynamic model of strategic disclosure in which a firm may privately receive information at a time that is random (and independent of the state of the economy). Because investors are uncertain regarding whether and when the firm has received information, the firm will not necessarily disclose the information immediately. We show that bad news about the economy can trigger the immediate release of information by firms. Conversely, good news about the economy can slow the release of information by firms. As a result, the release of negative information tends to be clustered. Surprisingly, this result holds only when firms can preempt the arrival of external information by disclosing their own information first. These results have implications for conditional variance and skewness of stock and market returns.
\end{abstract}

JEL classification: G14, G3, D82, M4

Keywords: disclosure, disclosure dynamics, strategic disclosure, disclosure timing, earnings announcement, stochastic volatility, skewness 


\section{Introduction}

One of the most important ingredients to the process of price discovery in financial markets is the flow of new information. The importance of information flow is perhaps most apparent during times of market "crisis," when it often seems that bad news is being reported simultaneously from multiple sources. This clustering of news could occur because firms learn more during bad times, or because firms strategically time the release of information. Indeed, it has long been recognized in the literature that corporate news disclosures are controlled by self-interested agents, and a number of theoretical and empirical analyses have found support for the idea that given this discretion, managers may choose to delay the release of bad news. ${ }^{2}$

As hinted above, in addition to delays in the release of information, casual observation suggests that disclosures of bad news are often clustered in bad times. While it is not surprising that firms' news are affected by market and sector conditions (given the correlation of their cash flows), the timing of the announcements is suggestive that these disclosure decisions are not made independently. Indeed, recent empirical work by Tse and Tucker (2007), who employ a duration model to study whether managers "herd" in announcing earnings warnings, finds that earnings warnings within an industry are clustered and that firms speed up their warnings in response to poor market conditions. In contrast, they show that such clustering is asymmetric in that good news does not generate such clustering.

In this paper, we seek an endogenous explanation for this asymmetry in the clustering of disclosure of good and bad news. We study disclosure dynamics when a firm possesses information that is correlated with market conditions, and explore managers' incentives to delay the disclosure of bad news, as opposed to good news, until market conditions worsen and become public knowledge. To the extent that such incentives exist, it can

\footnotetext{
${ }^{2}$ See for example Dye (1990), Rajan (1994), Dye and Sridhar (1995), Genotte and Trueman (1996), Shin (2003), Burgstahler and Dichev (1997),. Empirical support can be found in Miller (2002) who compares voluntary disclosures by firms that enjoy strong earnings performance to firms that experience earnings declines. He finds an increase in voluntary disclosures during periods of increased earnings.
} 
have important consequences for the aggregate information flow to the market and as a result for the conditional volatility and skewness of market and stock returns. In addition to their potential implications regarding market information flows and volatility, these results also suggest new empirical tests of whether managers do indeed time their information release.

We examine a dynamic game in which a manager of a firm decides when to report information regarding the value of the firm he manages. The manager maximizes the present value of her expected compensation, where the rate of compensation at each point in time is proportional to the market value of the firm conditional on public information. The firm learns its information at a random time. Because investors are uncertain whether a manager has learned the information, in equilibrium only those firms that have sufficiently positive news will release their information. Firms with more negative information will prefer to keep their market value higher - at least temporarily - by claiming that they do not yet have any information to report.

We then extend the model by supposing that in addition to the disclosure by the manager there is an external public signal about market conditions that will arrive at a future date. As alluded to earlier, while the timing of firm's information is uncorrelated with market conditions, we assume the value of the firm is correlated with this market news. Therefore, the public news announcement will affect the market value of the firm.

Our goal is to understand whether these interactions can lead to clustering in the release of information by firms even when the arrival of the underlying information is not clustered. To this end, we characterize the equilibrium conditions of this dynamic disclosure game and consider the resulting information release patterns. We begin in Section 3 with a negative, benchmark result. There we show that if the firm is unable to preempt the external news by releasing its own information ex-ante, then the external news has no effect on the firm's ex-post rate of disclosure. Thus, absent preemption there is no relation between the news announcement and the timing of disclosures.

Section 4 then establishes the main result of the paper. In contrast to the prior setting, we now allow the firm to release its information before the external news is announced. In the resulting dynamic disclosure game, because disclosure is irreversible, the firm faces a 
real options problem with regard to its disclosure decision: disclosing positive information may raise the stock price immediately, but gives up the option that the external public news would have had an even more positive impact on the stock price if the firm had not yet disclosed. In this case, we find that external market news can lead to an endogenous acceleration or deceleration in the rate of disclosure. In particular, in Proposition 5 -- the main result of the paper -- we show that the release of negative information about the market accelerates the release of information by the firm. Moreover, the particular pattern of acceleration depends on the level of the public news. We show that there are two regimes:

- If the information about market conditions is below a certain threshold then it may induce the firm to immediately release its information. The probability of immediate release is higher when the report on market conditions is lower.

- If the information about market conditions is above the threshold, then there is no immediate release of information by the firm. Still when we look at the delay until the report occurs, this delay is shorter when the level of the public news is lower.

One simple intuition for information clustering is the following: if the news about market conditions is bad, this will cause the market value of the firm to fall. This drop in value provides an incentive to release information if it is not as bad as the market now expects. That is, the release of negative external information lowers the threshold for disclosure, as the relative interpretation of the firm's news will become more favorable. However, the irrelevance results of Section 3 demonstrate that this simple intuition is not sufficient for the clustering of disclosures. For while negative news about market conditions does indeed lower the threshold for which the firm will disclose, it also lowers the posterior distribution of the firm's type. We show that generally, and somewhat remarkably, these effects perfectly cancel out and there is no clustering; that is, the probability that the firm will disclose is independent of the level of the public news about the economy. ${ }^{3}$

\footnotetext{
${ }^{3}$ Intuitively, the firm will only disclose its information if it is sufficiently good relative to the current expectations of investors. When there is good (bad) news, this news shifts upwards (downwards) both the distribution of the firm's signal and the expectations of investors. We show that these two effects offset each other, and thus there is no net increase in the probability of disclosure.
} 
Thus, the possibility of clustering emerges in our setting only if the firm has at least some likelihood of receiving its information prior to the arrival of external information and the opportunity to preempt the release of external information by disclosing its own signal first. The results of Section 4 show that clustering arises in a dynamic setting due to the endogeneity of the ordering of the disclosure decision. The key is that in such a setting, the distribution of types who have not yet disclosed when the public news about the state of the economy comes out is an endogenous subset of the original support. We show that in this case the threshold effects outweigh the distributional effects of the public news, and clustering emerges.

To summarize, our model illustrates that the timing option conferred upon firms in the dynamic disclosure game is critical to speeding up of disclosures when adverse news hits the market. Since positive correlation of types is required for these results, we conclude that bunching of disclosures is more likely within an industry, or more generally, within similar groups of firms.

In Section 5, we consider a number of extensions and further implications of our basic model. For example, we demonstrate that voluntary disclosures should cease in the period just prior to an anticipated public news announcement. We also discuss the case in which the "public news" the firm reacts to corresponds to voluntary disclosures by other firms in the industry. Finally, we show that our conclusions are robust to alternative specifications of the manager's payoff.

Section 6 of the paper discusses the implications of our results for stock returns. There we argue that while strategic disclosure leads to positive skewness of individual stock returns, the clustering effect leads to a higher correlation of returns in downturns. As a result, we can have negative skewness associated with the market index. Finally, the acceleration of disclosures after bad news implies that market volatility will increase after downturns. These implications find support in the existing evidence on the statistical properties of firm and market stock returns (see Black, 1976, Schwert, 1989, 1990, and Heston, 1993, among others). In contrast to the existing literature, our model provides an information-theoretic foundation for these stock return properties based on dynamic disclosure decisions of firms and managers. Finally, Section 7 concludes the paper. 


\section{Related Literature}

Our paper is related to a number of theoretical contributions concerning disclosure by firms. We explain below how our model differs from these models. In some cases, it is the fact that we examine multiple, correlated firms; in some others, it is our focus on a dynamic game; and, finally, in a few cases, the differentiating factor is the specific channel that drives our dynamic game with multiple firms.

Single-firm one-time disclosure models are considered in Shin (2003) and Shin (2006). Both papers consider disclosure by the manager of a firm with several independent projects. The information private to the manager is the number of projects whose uncertainty is resolved and whether the outcome was success or failure. The one-time disclosure consists of verifiable reports of some or all of these projects. The manager attempts to maximize the current share price and the markets rationally anticipate manager's disclosure policy. While this latter aspect of Shin's papers is similar to our setting, the disclosure in our model simply consists of revealing information at a given point of time or delaying its release; there is no possibility of any individual firm engaging in a partial disclosure at a given point of time as in Shin's papers. An equally important difference is that we examine the effect of external news on the firm's disclosure policy.

Static disclosure models with multiple firms that relate well to our model are found in Dye (1990) and Admati and Pfleiderer (2000). Both these papers consider firms with correlated information and model quality of disclosure as a (costly) choice of firms that affects the precision of disclosure. Dye considers risk-averse firm-owner and investor, and the relevant consideration is one of optimal risk-sharing between these agents at the

point of firm's sale. Admati and Pfleiderer, in contrast, have risk-neutral firm-owners and disclosures made by one firm are useful for investors in evaluating other firms (as in our model). They also assume that information asymmetry between firm-owners and investors reduces firm value. In both papers, the focus is on whether the voluntary disclosures by firms are at socially efficient levels of precision or not, and the primary result is that in equilibrium, firms may not internalize fully the externality (in risk-sharing or firm valuations) from their disclosure on other firms. Though the correlation structure 
of firm values is identical to our setup, the issue of delay in disclosure does not arise in these papers as there is only a one-time disclosure. ${ }^{4}$

Dynamic or sequential disclosure models are examined in Dye and Sridhar (1995), Gennotte and Trueman (1996), and Dierker (2002). Dye and Sridhar (1995), the most closely related paper out of these, considers a model with $n$ firms, each of which may or may not have privately observed a signal. If a firm gets a signal it can disclose it in period 1 or 2 . These features of their model are similar to ours. However, they assume that whether firms observe a signal or not is positively correlated, but the signals themselves are independent. This is exactly the dual of our assumption that signals are correlated but their arrival process is independent. As a result, in their model, it is more disclosures in period 1 that leads to more disclosures in period 2 (since investors believe that nondisclosing firms have adverse information), whereas in our model, it is the nature of disclosure (good news or bad news) that delays or triggers disclosure by other firms. Hence, a key difference is that they assume the information arrival process is itself correlated. In contrast, in our model clustering of disclosures is due only to the strategic element and in particular not due to the correlated arrival of information. Another crucial difference between their results and ours is that in our model, clustering is not symmetric in quality of news. Specifically, when information arrival is correlated as in Dye and Sridhar, there is no difference in clustering of good news or bad news; in contrast, when information is correlated but not its arrival as in our model, there is clustering of bad news but not of good news.

Dierker's single-firm, dynamic disclosure game is different from our model in the managerial objective (making the market as informationally efficient as possible), the benefit of disclosing early (providing information to firm's early customers), and the cost of disclosing early (discouraging information production by investors). Gennotte and Trueman also consider a single-firm model where the focus is on timing of mandated disclosures (for instance, should earnings be disclosed intra-day or outside of trading

\footnotetext{
${ }^{4}$ Rajan (1994) examines a slightly different model in which banks coordinate on disclosure of their news. If state of the economy is good, then banks may hide their bank loans by extending further credit. In contrast, if the underlying state is bad, then banks reveal their losses. This result is generated in Rajan's model through the assumptions that managers are short-termist and that in the bad state, the distribution of managerial types collapses to a common one.
} 
hours). They examine whether multiple pieces of information reaching a firm should be bunched together in a disclosure or disclosed sequentially. In contrast, our focus is on whether information reaching a firm may be released in equilibrium in a bunched manner with external market information on the industry or the economy. ${ }^{5}$

Most of the papers discussed above, including ours, assume that managerial payoff is linear in market's best expectation of manager's type. Aboody and Kasznik (2000) show empirically that disclosure decisions are also affected to an extent by option-like payoffs for CEOs. Incorporating non-linear payoffs of this type should not take away much from the intuition of our results, but it is difficult to analytically characterize solutions in their presence. Finally, we do not focus in our model on the incentives of investors to collect information (as in, Diamond 1985). That is, information reaching the markets in our model is entirely controlled by disclosure decisions of managers.

\section{Benchmark I- Strategic Disclosure without External News}

In this section we develop a benchmark model of strategic disclosure by a firm in the absence of any external news. Our model builds on the static model of Dye (1990), in which investors are unsure whether the manager has learned new information. This uncertainty allows managers with bad news to claim that they have no new information, rather than disclose it. As a result, only firms with sufficiently good news will reveal their information. We then consider a dynamic version of the model. There we show that firms with bad news will delay their disclosure, and that the length of the delay is longer for worse news.

\footnotetext{
${ }^{5}$ Teoh and Hwang (1991) consider a model wherein two types of firms ("high" and "low" types) and two kinds of information that firms wish to disclose ("news" which if disclosed is verifiable and "type" which is not verifiable). Hence, the disclosure decisions are driven by signalling considerations and have the feature that high types withhold good news and reveal bad news and low types do the converse. In contrast, our model has only type-related information, which if disclosed, is verifiable. Each piece of information in Teoh and Hwang is private only for a period, though their arrival is sequential, and this lends their model a certain dynamic aspect, even though it is quite different from ours.
} 


\subsection{A Model of Partial Disclosure}

Consider a single firm whose manager may learn some information relevant to the firm's value. Let the manager's signal $S$ be the value of the firm conditional on this new information. ${ }^{6}$ The manager learns this information with probability $p \in[0,1]$. Once the manager is informed, with probability $q$ both the manager's signal and the fact that the manager is informed are private information. When privately informed, the manager has discretion regarding the release of the information: The manager may either disclose it or conceal it, but if it is disclosed it is verifiable and cannot be manipulated. ${ }^{7}$ With probability $1-q$ the manager is "publicly" informed and so does not have discretion over the release of information and reports it immediately to the market. ${ }^{8}$ The distribution of the signal $S$ is independent of whether the manager becomes informed or has discretion. For convenience, we assume that $S$ is non-degenerate and continuously distributed on some (possibly unbounded) interval.

The manager's objective is to maximize the firm's market value, which is its value conditional on the information available to investors. Because the signal $S$ is the firm's expected value, if the manager discloses the signal the firm's market value will simply be $S$. If the manager does not disclose, the firm's market value will be a fixed amount, $v$, based on the information contained in the fact that the manager did not disclose. Because the benefit from disclosing is increasing in the manager's signal, the equilibrium disclosure policy will follow a threshold rule: only firms with a signal higher than some threshold $x^{*}$ will disclose their information. Naturally, the manager is willing to disclose $S$ if and only if it exceeds the value the firm would have without disclosing, and so the manager will disclose if

$$
S \geq x^{*}=v \equiv E[S \mid \text { nondisclosure }]
$$

Consider first the case in which it is public knowledge that the manager is informed (i.e., $p=1$ or $q=0$ ), but investors do not know the manager's information. In that case the

\footnotetext{
${ }^{6}$ This assumption is essentially without loss of generality and is equivalent to assuming the manager learns information $I$ and the firm's value is $V$, and then defining $S=E[V \mid I]$.

${ }^{7}$ This assumption of "verifiable reports" is common to the literature. See, for example, Shin $(2003,2006)$.

${ }^{8}$ This case captures situations in which either (i) the information itself is public, (ii) the fact that the manager has learned the information is public (in which case immediate full disclosure will occur in equilibrium), or (iii) hiding the information would be too costly (perhaps due to legal concerns).
} 
manager would not disclose only if $S \leq x^{*}$, so that from (1) the equilibrium threshold satisfies

$$
x^{*}=E\left[S \mid S \leq X^{*}\right]
$$

This equation has the unique solution that $x^{*}$ is the minimum of the support of $S$, and all information is disclosed. This result replicates the standard "unraveling" result - noted, e.g., by Ross (1979), Grossman (1981), Milgrom (1981) and others - that if the market knows for sure that a firm holds some information then in equilibrium the firm will disclose its information.

However, with $p<1$, investors do not know if the manager is informed. In that case the payoff in the event of non-disclosure can be calculated as

$$
\begin{aligned}
E[S \mid \text { nondisclosure }] & =E\left[S \mid\left(\begin{array}{c}
\text { manager is } \\
\text { uninformed }
\end{array}\right) \text { or }\left(\begin{array}{c}
\text { manager is privately informed without } \\
\text { the market knowing this and } S \leq x^{*}
\end{array}\right)\right] \\
& =\frac{(1-p) E[S]+p q \operatorname{Pr}\left(S \leq x^{*}\right) E\left[S \mid S \leq x^{*}\right]}{(1-p)+p q \operatorname{Pr}\left(S \leq x^{*}\right)}
\end{aligned}
$$

Hence we can write the equilibrium condition for the threshold $x^{*}$ as

$$
x^{*}=h_{S}\left(x^{*}, \rho\right) \equiv \frac{E[S]+\rho \operatorname{Pr}\left(S \leq x^{*}\right) E\left[S \mid S \leq x^{*}\right]}{1+\rho \operatorname{Pr}\left(S \leq x^{*}\right)}
$$

where $\rho \equiv \frac{p q}{1-p}$ is the relative likelihood that the manager is privately informed versus uninformed.

Equation (3) expresses the equilibrium threshold as a fixed point of the function $h$. While the precise solution to (3) will depend on the distribution of $S$, we have the following useful characterization, which generalizes the intuition that in the absence of disclosure, investors will adopt the "worst case beliefs":

Proposition 1. Equation (3) has a unique solution $x^{*}$ which is the equilibrium disclosure threshold. This threshold is decreasing with $\rho$ and satisfies

\footnotetext{
${ }^{9}$ See appendix for all proofs not in the text.
} 


$$
x^{*}=\min _{x} h_{S}(x, \rho)
$$

This disclosure policy yields the lowest possible value of the firm in the event of non-disclosure of any policy (threshold or not).

The proposition implies that the equilibrium is well defined and unique. Not surprisingly, the amount of disclosure increases with the likelihood that the manager is privately informed. Equation (4) can be interpreted as generalization of the intuition from the standard full-disclosure equilibrium in (2): Investors interpret non-disclosure as pessimistically as possible, so that the equilibrium threshold is the one that leads to the lowest value for the firm in the event of non-disclosure. Figure 1 depicts the calculation of the equilibrium threshold.
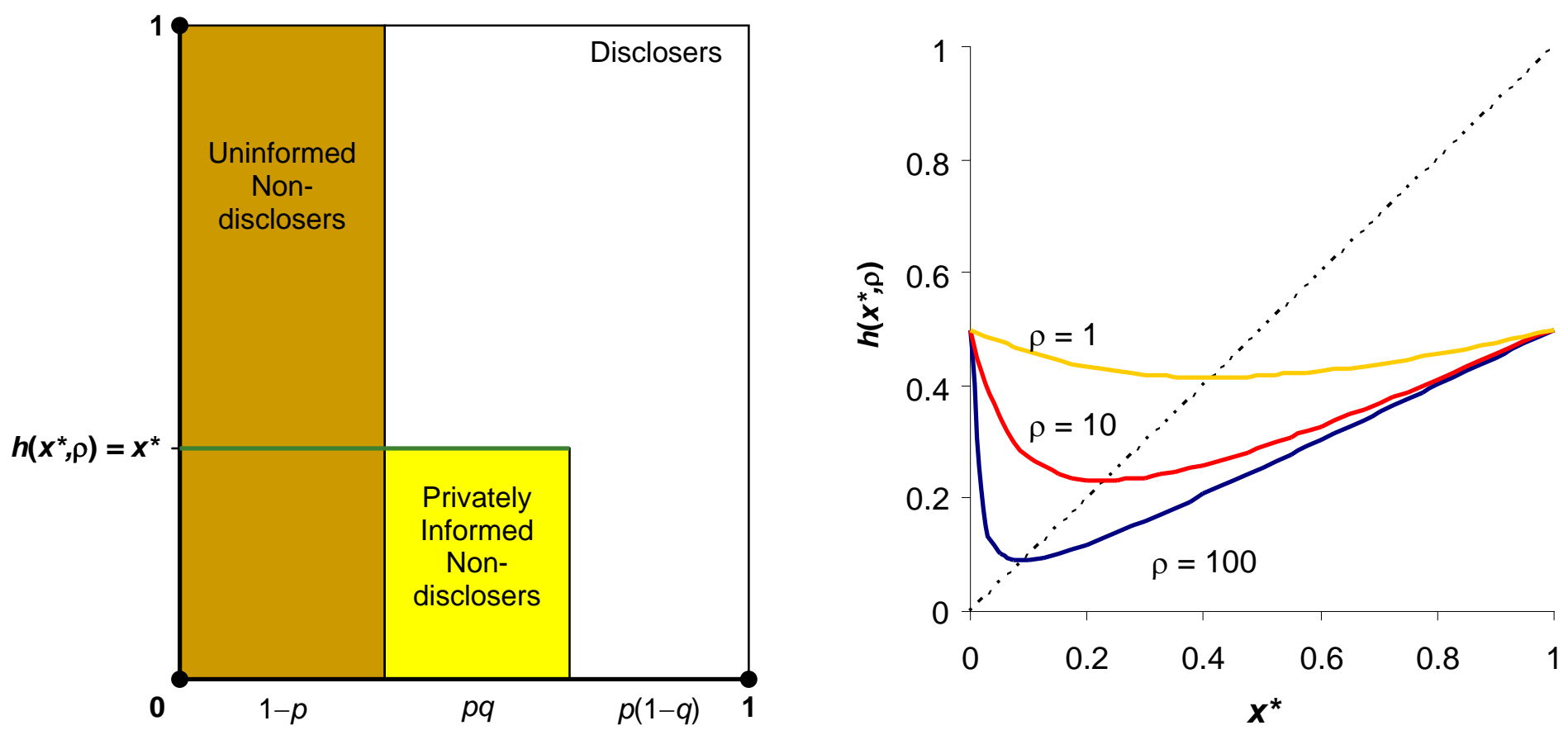

Figure 1: Calculation of Disclosure Threshold

The left panel illustrates the disclosure threshold given the equilibrium set of non-disclosing and disclosing firms in the case when $S$ is uniform on [0,1]. The right panel plots $h\left(x^{*}, \rho\right)$ for $\rho=1,10$, and 100, confirming that the fixed point occurs at the minimum of the function $h$. 
Given the equilibrium disclosure threshold $x^{*}$, we can compute the probability of disclosure as

$$
\pi=p\left(1-q+q \operatorname{Pr}\left(S>x^{*}\right)\right)
$$

Not surprisingly, this probability increases with the probability $p$ that the manager is informed. Also, because $x^{*}$ declines with $p$, strategic effects imply that the rate of increase in $\pi$ exceeds that in $p$.

\subsection{Dynamic Disclosure and Delay}

We now consider a dynamic version of the disclosure game in the previous section. The setting is a continuous-time game for time $t \in[0, T]$. The probability that a manager is informed by time $t$ is given by a non-decreasing function $p(t)$. Again we assume that when the manager becomes informed there is a probability $q$ that the manager has discretion regarding when to disclose the signal $S$ to the market.

Let $I_{t}$ be the information that is public at time $t$. Then the market value of the firm on date $t$ is given by $v_{t}=E\left[S \mid I_{t}\right]$. We assume the manager's payoff is increasing in the market value of the firm at any moment in time. The exact form of this payoff will not affect the qualitative results, and so for simplicity we represent the payoff to the manager of firm $i$ as

$$
\int_{t=0}^{T} \lambda(t) u\left(v_{t}\right) d t
$$

where $u$ is increasing and_the weights $\lambda(t)>0$ may reflect, e.g., discounting or fluctuations in the sensitivity of the manager's wage to the share price. (Again, we may think of the manager's compensation as based on his perceived ability, which varies linearly with the firm's market value.) We assume the weights $\lambda(t)$ are bounded above.

What is the optimal disclosure policy for the manager in this setting? If the firm discloses its signal then all information will be revealed and its market value will remain equal to its signal $S$ from that point onward. If the firm does not disclose, its market value will only depend on the fact that it has not yet disclosed. Thus, the benefit from disclosing is increasing in the signal $S$, whereas the payoff from not disclosing does not 
depend on $S$. Therefore, the manager's optimal disclosure strategy at date $t$ can again be described by a threshold, which we denote by $x^{*}(t)$.

Because the likelihood the firm is informed, $p(t)$, increases over time, the results of Section 2.1 suggest that if the firm does not disclose, investors will become more pessimistic over time regarding its information. In that case, the market value of the firm will decline over time if there is no disclosure. The manager will therefore voluntarily disclose if and only if the signal $S$ exceeds the firm's current market value absent disclosure. As this condition is the same equilibrium condition as in Section 2.1, we can combine it with the results of Proposition 1 to characterize the equilibrium disclosure threshold as follows:

Proposition 2. Let $\rho(t) \equiv \frac{p(t) q}{1-p(t)}$. Then the decreasing disclosure threshold,

$$
x^{*}(t)=\min _{x} h_{S}(x, \rho(t))=h_{S}\left(x^{*}(t), \rho(t)\right)
$$

is the unique equilibrium of the dynamic disclosure game.

We can compute the rate of disclosure in this dynamic equilibrium by calculating the cumulative disclosure at each date using (5). The next result considers the effect of changing the mean or variance of the firm's signal on the equilibrium disclosure rate:

Corollary A. Suppose $\tilde{S}=a+b S$ with $b>0$. Then if $x^{*}(t)$ is the equilibrium threshold for signal $S$, the equilibrium threshold for signal $\tilde{S}$ is given by

$$
\tilde{x}^{*}(t)=a+b x^{*}(t)
$$

Thus, the probability of disclosure is independent of the parameters $a, b$.

Proof of Corollary A: The result follows immediately from the fact that $h_{\tilde{S}}(a+b x, \rho)=a+b h_{S}(x, \rho) \quad$ It can be verified that $\tilde{x}^{*}(t)=a+b x^{*}(t)$ is a solution to equation (3) with $\tilde{S}=a+b S$. Iqed 
COROLlary A has an important, perhaps surprising, implication: the equilibrium level of disclosure will not depend on either the mean or the variance of the manager's signal. We shall exploit this property below, when we consider the impact of outside information on the manager's disclosure decision. See Figure 2 for an illustration of the equilibrium when the manager's signal is normally distributed.
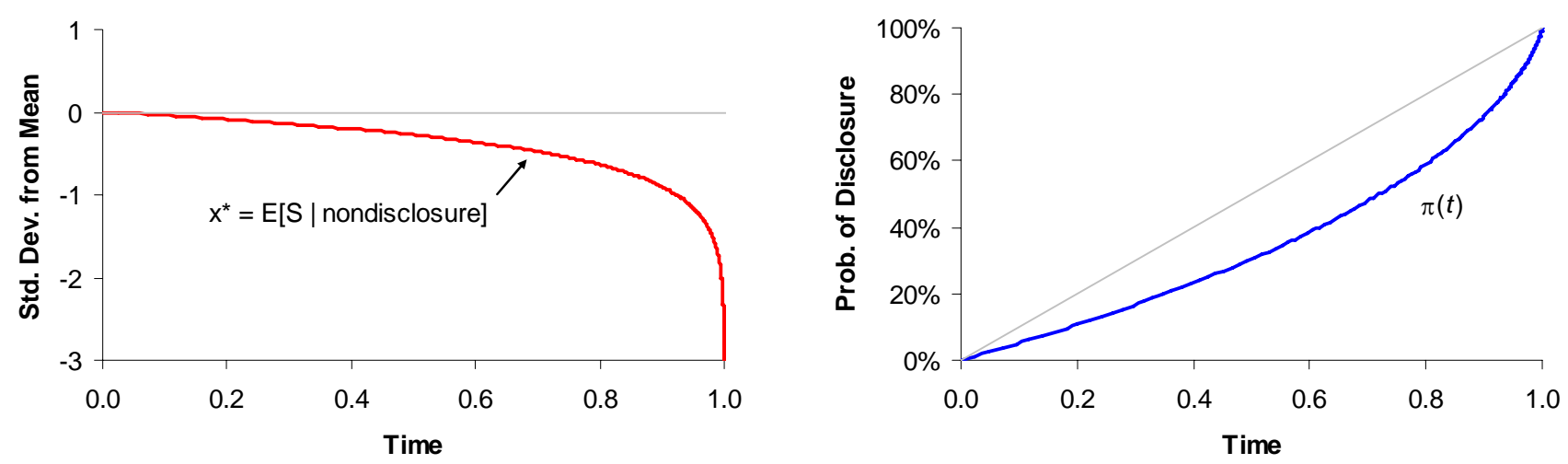

Figure 2: Dynamic Disclosure Threshold and Probability

The left panel shows the equilibrium disclosure threshold as a function of $t$ given $p(t)=t, q=0$, and $S$ is normally distributed. The right panel shows the cumulative probability of disclosure, where it is evident that $p(t)$ first-order stochastic dominates $\pi(t)$.

\section{Benchmark II- External News without Preemption: An Irrelevance Result}

In this section we consider the impact of external news which is announced prior to the firm's disclosure decision. We assume that the firm can disclose only after the external news is released.. We will show that under fairly general conditions, the announcement of prior news will have no impact on the firm's disclosure rate. This result will serve as a useful benchmark when we consider the possibility of preemptive disclosure by the firm in the next section.

\subsection{A Model with External News}

Consider an external news event that is informative regarding the firm's private signal and market value. This event may correspond to public news about the firm or its industry, or the disclosure of private information by another firm with correlated cash 
flows. Suppose this external news is released prior to the firm having any opportunity to disclose its information.

Intuitively, a negative news announcement will cause the market value of the firm to decrease. Thus, holding fixed the manager's signal $S$, negative news will make it more likely that the manager will disclose this information. Despite this intuition, however, we show in this section that if the news $Y$ is released before the firm has the opportunity to reveal $S$, then there is no impact of the news $Y$ on the rate of disclosure; i.e., there is no disclosure "clustering" after bad news in this case.

To capture this correlation between the news and the firm's signal, we denote the news announcement by the random variable $Y$, and suppose the firm's signal $S$ and the news $Y$ are jointly normally distributed with a non-zero correlation. ${ }^{10}$ The news $Y$ is announced at time 0 , before the firm has an opportunity to disclose its information $S$. How does the announcement of $Y$ affect the firm's disclosure strategy?

In this setting, the firm's equilibrium disclosure threshold will depend on both the realization of the news $y$ and the probability that it is informed $p(t)$, and so can be written as $X *(Y, t)$. Because $S$ and $Y$ are joint normal, we can without loss of generality write

$$
S=a+\beta Y+\sigma \varepsilon
$$

where $\varepsilon$ is a standard normal random variable and $Y$ and $\varepsilon$ are independent. We assume (again without loss of generality) that $\beta>0$, so that "bad news" corresponds to a low realization of $Y$. Therefore, the announcement of the news $Y$ will affect the mean of the posterior distribution of $S$, and also reduce its conditional variance compared to its unconditional variance.

\subsection{Independence of Equilibrium Disclosure Rates}

From (9) we can see that the news announcement $Y$ changes the posterior distribution of the signal $S$ according to a linear transformation of the normal distribution. Therefore, we can immediately apply COROLLARY A to characterize the equilibrium:

\footnotetext{
${ }^{10}$ For simplicity, we assume that firm's signal $S$ is a sufficient statistic for the firm's value (and the manager's payoff) given the news $Y$. For a more general setting in which $Y$ may also have an effect on the manager's payoff, see Section 5.4.
} 
Proposition 3. Given the news announcement $Y$ at date 0 , there exists a function $z *(t)$ such that the equilibrium disclosure threshold for the firm is given by $^{11}$

$$
x_{n p}^{*}(Y, t)=a+\beta Y+\sigma Z^{*}(t)
$$

The probability that the firm discloses by date $t$ is independent of the news $Y$ and is given by

$$
\pi(t)=p(t)\left[1-q+q\left(1-N\left(z^{*}(t)\right)\right)\right]
$$

where $N$ is the standard normal distribution function. This disclosure probability is identical to the firm's disclosure probability if the news $Y$ were not revealed.

Proof of Proposition 3: Let $z *(t)$ be the unique solution to $z *=h_{\varepsilon}(z *, \rho(t))$. Because the conditional distribution of $S$ can be expressed as a linear transformation of $\varepsilon,(10)$ and (11) follow directly from Corollary A. By the same logic, if the information $Y$ were not revealed, $S$ would have an equivalent distribution to $\mu_{S}+\sigma_{S} \varepsilon$, and the probability of disclosure would again by given by (11). Iqed

As one might expect, Proposition 3 demonstrates that the firm's disclosure threshold is affected by the prior news announcement. Bad news (i.e., news such that $\beta Y<0$ ) will lower the threshold. Thus, for a given signal $S$, the firm will disclose more quickly in the event of bad news.

However, while the disclosure threshold is lower given bad news, the distribution of the firm's signal is also lower. We can see by comparing (9) and (10) that these two effects offset each other, and in the end there is no impact on the probability of disclosure, as shown in (11).

Thus we have the striking implication that, if the firm cannot preempt the external news announcement by disclosing its own information first, there will be no clustering effect. That is, the probability of disclosure by the firm will not be affected by prior news

\footnotetext{
${ }^{11}$ The notation "np" stands for no-preemption, to contrast the results here with those in the next section.
} 
announcements. Intuitively, the firm will only disclose its information if it is sufficiently good relative to the current expectations of investors. When there is good news, this news shifts upwards both the distribution of the firm's signal and the expectations of investors. These two effects offset each other, and thus there is no net increase in the probability of disclosure.

Remark. While the results in this section were derived using a normal distribution, they are clearly more general. Suppose, for example, that $S$ and $Y$ are jointly log-normal. Then we can decompose $S$ as $S=a Y^{\beta} \varepsilon$ for some $\beta$ where $\varepsilon$ is log-normal and independent of $Y$. The same argument again implies that the realization of $Y$ will have no effect on the rate of disclosure. Indeed, the result obtains in any setting for which the posterior distribution of $S$ can be expressed as $S=\mu(Y)+\sigma(Y) \varepsilon$.

\section{Preemption and Disclosure Clustering}

In this section, we change the setting of Section 3 by allowing the firm to disclose early and preempt the news announcement $Y$. We then develop the main result of the paper: We show in Proposition 5 below that, when preemption is possible, disclosures will be clustered after the announcement of bad news. Indeed, a sufficiently negative news announcement can trigger an immediate disclosure of the firm's information, and will accelerate the rate of disclosure if the firm delays.

To show this result, we proceed as follows. First we extend the current model to allow for the possibility that the firm may receive information prior to the arrival of public news in Section 4.1. Section 4.2 then characterizes disclosure policies following the arrival of public news provided that the firm did not previously disclose its information; Section 4.3 formalizes the disclosure decision prior to the arrival of public news, that is, under what conditions should the firm preempt public news with its disclosure; and, finally, Section 4.4 derives our main result (Proposition 5) on the effect of public news on disclosure rate. 


\subsection{A Model with Preemption}

Specifically, the model we consider is identical to the setting in Section 3 except that the public news announcement $Y$ occurs at date $t=1$ rather than $t=0 .{ }^{12}$ Thus, by disclosing its signal $S$ in the interval $t \in[0,1)$, the firm can preempt the news announcement.

How does the opportunity to preemptively disclose $S$ change the equilibrium rate of disclosure before and after the announcement of the news $Y$ ? For simplicity, consider the case in which the manager is initially informed with probability $p(0)$ and the manager receives no new information between dates 0 and 1 . That is

$$
p(t)=p(0) \text { for all } t \in[0,1]
$$

The assumption in (12) implies that all disclosures that take place prior to date 1 will occur at date 0 . Intuitively, as nothing changes between date 0 and 1 , a firm that would disclose prior to date 1 will do so immediately. This assumption simplifies the analysis of the preemption stage to a single date 0 disclosure threshold, $x^{*}(0)$. We will discuss a setting with $p(t)$ increasing between date 0 and 1 in Section 5.1 .

\subsection{Post-News Disclosure Policies}

We begin our analysis by assuming that the manager follows an initial disclosure threshold $x^{*}(0)$ prior to the news announcement, and solving for the equilibrium disclosure threshold $x^{*}(Y, t)$ on date $t \geq 1$ after the news announcement $Y$ at date 1 .

Suppose that $x^{*}(Y, t)$ is weakly decreasing with $t$ (we will verify this shortly). Then if a firm has not disclosed by date $t$, there are three possibilities:

i) the manager was uninformed up to date $t$,

ii) the manager was privately informed before date 1 , and $S \leq \min \left(x^{*}(0), x^{*}(Y, t)\right)$,

iii) the manager was privately informed between date 1 and $t$, and $S \leq x^{*}(Y, t)$.

Given these alternatives, we can compute the market value of the firm given nondisclosure on date $t$ as the expected value of $S$ conditional on $Y$ and (i)-(iii). As in

\footnotetext{
${ }^{12}$ We consider the case in which the date of the news announcement is uncertain in Section 5.2.
} 
Section 2.2, the equilibrium disclosure threshold will equal this market value; the manager will not choose to disclose unless doing so will improve the firm's share price relative to not disclosing.

To compute the equilibrium, note that if $x^{*}(Y, t) \leq x^{*}(0)$, then conditions (ii) and (iii) above can be combined as

ii') the manager was informed before date $t$, and $S \leq x^{*}(Y, t)$.

Condition (ii') is precisely the same information available in the equilibrium in the prior Section 3. Therefore, the equilibrium threshold coincides with the equilibrium policy $x_{n p}^{*}(Y, t)$ of Section 3.1, and we have

$$
x^{*}(Y, t)=a+\beta Y+\sigma z^{*}(t) \text { if } Y \leq y_{0}(t)
$$

where $y_{0}(t)$ is defined as the critical value such that $a+\beta y_{0}(t)+\sigma z^{*}(t) \equiv x^{*}(0)$. Note that we can also interpret the condition $Y \leq y_{0}(t)$ as $t \geq t_{0}(Y)$ for some $t_{0}$ increasing with $Y$; that is, for any given $Y$, the post-news policy will eventually coincide with the no-preemption policy.

Next suppose $x^{*}(Y, t)>x^{*}(0)$, so that conditions (ii) and (iii) are stronger than (ii'). In this case, the set of non-disclosing firms excludes firms that were informed before date 1 with signals between $x^{*}(0)$ and $x^{*}(Y, t)$. Because we are excluding firms whose signal is below the average $x^{*}(Y, t)$, this raises the average quality of the pool of non-disclosing firms compared to condition (ii'). Therefore, we have shown that

$$
x^{*}(Y, t)>a+\beta Y+\sigma Z^{*}(t) \text { if } Y>y_{0}(t)
$$

Moreover, the above intuition suggests that the gap between $x^{*}(Y, t)$ and $a+\beta Y+\sigma z^{*}(t)$ increases with the gap between $Y$ and $y_{0}(t)$. The following result formalizes this intuition:

Proposition 4. If the firm can preemptively disclose its signal $S$ at date 0 prior to the news announcement $Y$ at date 1 , the equilibrium disclosure threshold at date $t \geq 1$ is given by

$$
x^{*}(Y, t)=a+\beta Y+\sigma Z^{*}(t)+k(Y, t)
$$


where $k(Y, t)=0$ if $Y \leq y_{0}(t)$, and $k(Y, t)>0$ and strictly increasing in $Y$ for $Y>y_{0}(t)$. The threshold $x^{*}(Y, t)$ strictly decreases with $p(t)$.

We illustrate the results of Proposition 4 in Figure 3. After the news $Y$ is announced, the disclosure policy $x^{*}$ coincides with the no-preemption policy $x_{n p}^{*}$ except when $x_{n p}^{*}$ exceeds the initial threshold $x^{*}(0)$. In that case, $x^{*}$ exceeds $x_{n p}^{*}$.

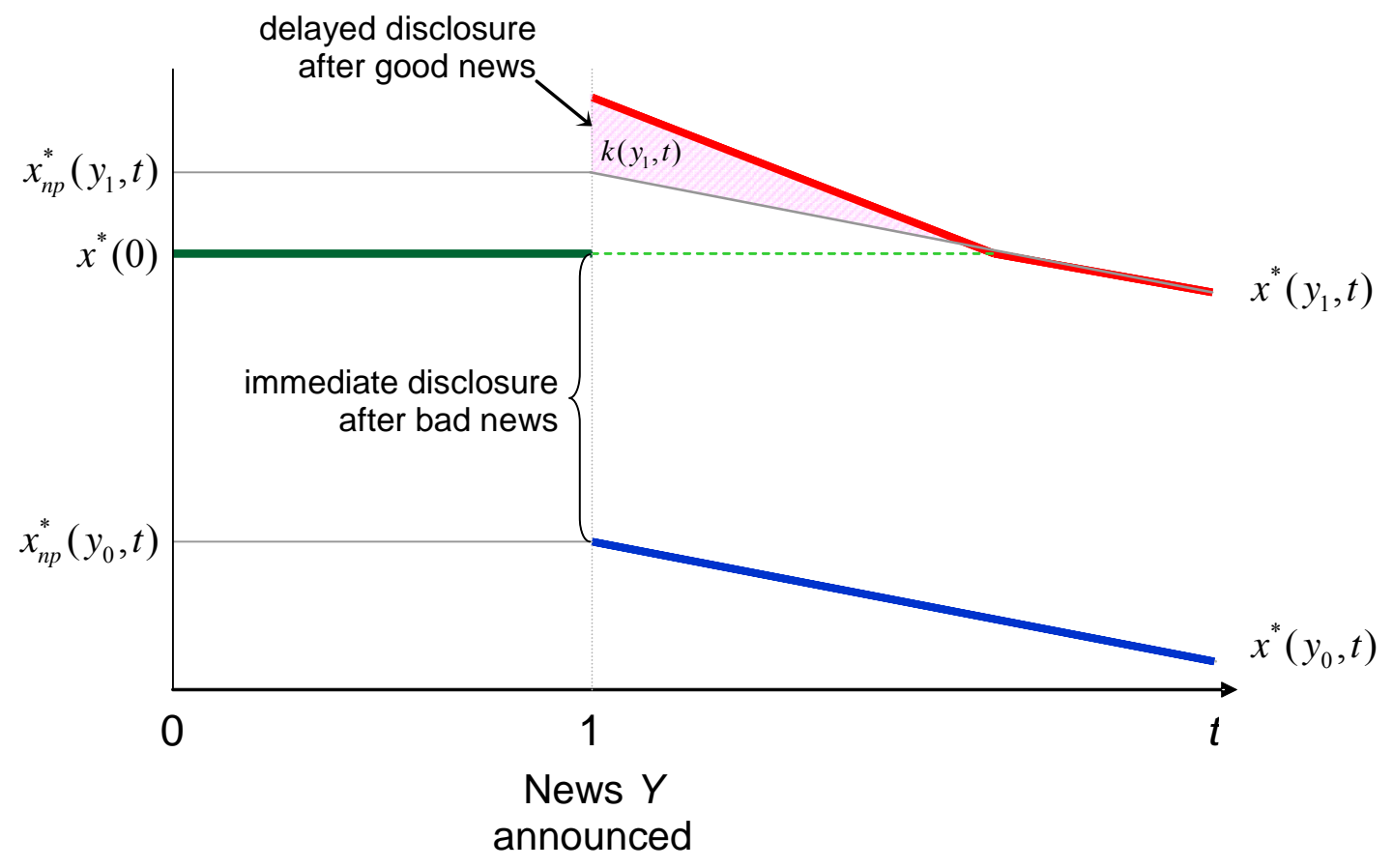

Figure 3: Equilibrium Post-News Disclosure Policies

The figure shows the equilibrium post-news disclosure thresholds. These thresholds coincide with the nopreemption thresholds when they are below $x^{*}(0)$. When above $x^{*}(0)$, they exceed the no-preemption thresholds by $k(Y, t)$.

\subsection{Preemptive Disclosure}

Now that we have characterized the optimal disclosure policy for the firm once the news $Y$ has been released at date 1 , we consider next the equilibrium level of preemptive disclosure at date 0 . Let $v(0)$ be the market value of the firm at date 0 if there is no 
disclosure. If the manager discloses if and only if the signal $S$ exceeds a threshold $x *(0)$, then from the analysis in Section 2,

$$
v(0)=h_{S}\left(x^{*}(0), \rho(0)\right)
$$

In our prior analysis, we used the equilibrium condition that the manager would disclose if the signal $S$ exceeded the firm's market value. In the case of preemptive disclosure, however, a real option problem emerges. If a firm with signal $S$ discloses at date 0 , then the market value of the firm will equal $S$ from that point onward. If it does not disclose at date 0 , there is a chance that the news $Y$ will be sufficiently positive that its market value will exceed $S$ for some time if it delays disclosure. Disclosing at date 0 forfeits this option. Thus, the firm will not disclose at date 0 unless its immediate gain from disclosing exceeds that value of this option.

Formally, the immediate gain from disclosing at date 0 is $u(S)-u(v(0))$. Because the market value of a non-disclosing firm on date $t \geq 1$ is $x^{*}(Y, t)$, the potential gain from not disclosing at date $t$ is $\left(u\left(x^{*}(Y, t)\right)-u(S)\right)^{+}$. Then, from (6), a firm with signal $S$ prefers to disclose if

$$
\int_{0}^{1} \lambda(t)[u(S)-u(v(0))] d t>E\left[\int_{1}^{\infty} \lambda(t)\left[u\left(x^{*}(Y, t)\right)-u(S)\right]^{+} d t \mid S\right]
$$

If we normalize $\int_{0}^{1} \lambda(t) d t=1$, then the equilibrium disclosure threshold satisfies ${ }^{13}$

$$
x^{*}(0)=u^{-1}\left(u(v(0))+E\left[\int_{1}^{\infty} \lambda(t)\left[u\left(x^{*}(Y, t)\right)-u(S)\right]^{+} d t \mid S=x^{*}(0)\right]\right)
$$

We can use (16) and (18), together with the analysis in Proposition 4 to characterize the equilibrium disclosure policy in this setting.

\footnotetext{
${ }^{13}$ Guaranteeing the optimal strategy is indeed a threshold strategy requires showing that if (17) holds for $S$, then it holds for all $S^{\prime}>S$. In the appendix we prove this for the case $u(x)=x$ and $\sigma>\frac{1}{2} \beta \rho_{0}^{1 / 2}$, which puts an upper bound on the informativeness of the news. This condition, however, is by no means necessary; e.g., in the extreme alternative case $\sigma=0$, it is easy to see that a threshold strategy is optimal as $Y$ is then a perfect signal of $S$ and the option value of waiting disappears. As a practical matter, we can simply solve for $x^{*}(0)$ assuming a threshold exists, and then verify that it is optimal ex post (which is the case for all examples we have considered).
} 


\subsection{Disclosure Rates and Clustering}

Now that we have characterized the equilibrium, we examine the impact of the news $Y$ on equilibrium rate of disclosure. Looking again at Figure 3, we can see the following intuitive implications: First, if the public news is sufficiently poor $\left(Y<y_{0}(1)\right)$, then $x *(Y$, $1)<X^{*}(0)$. Thus, if the manager is privately informed with a signal in that range, the firm will disclose its information immediately following the public news. Second, if the news is sufficiently good $\left(Y>y_{0}(1)\right)$, then the optimal disclosure threshold is distorted upward by $k(Y, 1)$, which delays the rate of future disclosures. We formalize both of these observations in the remainder of this section.

Given news $Y$, the rate of pre-emptive disclosure at date 0 is given by

$$
\pi(0 \mid Y)=p(0)\left[1-q+q \operatorname{Pr}\left(S \geq x^{*}(0) \mid Y\right)\right]
$$

Next, the probability of disclosure by date $t \geq 1$, given the news $Y$, is given by

$$
\begin{aligned}
\pi(t \mid Y)= & p(0)\left[1-q+q \operatorname{Pr}\left(S \geq x^{*}(0) \wedge x^{*}(Y, t) \mid Y\right)\right] \\
& +[p(t)-p(0)]\left[1-q+q \operatorname{Pr}\left(S \geq x^{*}(Y, t) \mid Y\right)\right]
\end{aligned}
$$

Our main interest is in the rate of disclosure in the interval between the news announcement at date 1 and a future date $t \geq 1$, we denote by

$$
\Delta \pi(t \mid Y) \equiv \pi(t \mid Y)-\pi(0 \mid Y)
$$

\section{Immediate Disclosure}

Consider first $\Delta \pi(1 \mid Y)$, which is the probability that a firm discloses its signal immediately upon the announcement of the news $Y$. Because $p(1)=p(0)$, absent the news announcement there would be no disclosures at date 1. Thus, if $\Delta \pi(1 \mid Y)>0$, there is a positive probability that the news announcement will "trigger" a disclosure.

We can evaluate

$$
\Delta \pi(1 \mid Y)=p(0) q \operatorname{Pr}\left(x^{*}(0) \geq S \geq x^{*}(Y, 1) \mid Y\right)
$$


Thus, the news announcement $Y$ can trigger a disclosure if $x^{*}(Y, 1)<x^{*}(0)$. From Proposition 4, immediate disclosure can occur if $Y<y_{0}(1)$. In that case,

$$
\Delta \pi(1 \mid Y)=p(0) q\left[N\left(\left(x^{*}(0)-a-\beta Y\right) / \sigma\right)-N\left(z^{*}(t)\right)\right]
$$

which strictly decreases with $Y$.

\section{Accelerated Disclosure}

Consider now the rate of disclosure in a window of time after the news announcement. Specifically, we look at the likelihood of a disclosure between the time of the news announcement and a future date $t>1$ with $p(t)>p(0)$. In this case, additional disclosures will occur by firms who learn their information after the news was released. We show that these disclosures create an additional dependence of the disclosure rate on the news $Y$.

From (19)-(21), we have

$$
\Delta \pi(t \mid Y)=p(0) q \operatorname{Pr}\left(x^{*}(0) \geq S \geq x^{*}(Y, t) \mid Y\right)+[p(t)-p(0)] q \operatorname{Pr}\left(S \geq x^{*}(Y, t) \mid Y\right)
$$

Let us analyze each of the probabilities in (24) separately. First, from the results of Proposition 4,

$$
\operatorname{Pr}\left(x^{*}(0) \geq S \geq x^{*}(Y, t) \mid Y\right)=\left[N\left(\left(x^{*}(0)-a-\beta Y\right) / \sigma\right)-N\left(z^{*}(t)\right)\right]^{+}
$$

This expression is strictly decreasing in $Y$ for $Y<y_{0}(t)$.

The second probability in (24) reflects disclosures by firms newly informed during the period between date 1 and date $t$. Again using the results of Proposition 4,

$$
\operatorname{Pr}\left(S \geq x^{*}(Y, t) \mid Y\right)=\left[1-N\left(z^{*}(t)+k(Y, t) / \sigma\right)\right]
$$

Recall that $k(Y, t)$ is increasing in $Y$, and therefore this probability is decreasing, in $Y$ for $Y$ $>y_{0}(t)$.

Combining these results leads to our main result of the paper, relating the rate of disclosure with the quality of the public news announcement: 
Proposition 5. For any date $t>1$ after the public news announcement, the probability of disclosure $\Delta \pi(t \mid Y)$ during after the period $[1, t]$ is strictly decreasing in $Y$ throughout the support of $Y$. If $Y<y_{0}(1)$, there is a positive probability, which is higher if $Y$ is lower, of an immediate discretionary disclosure at date 1 , followed by a disclosure rate that does not depend on $Y$. If $Y>y_{0}(1)$, there is no immediate discretionary disclosure at date 1 , and the rate of subsequent disclosures decreases with $Y$.

\section{Extensions of the Model}

\subsection{Continuous Preemption and Information Blackouts}

In the basic model in Section 4, the firm either learns its signal at date 0 or after the news announcement on date 1. Here we consider what happens if the firm's signal has a positive arrival rate between dates 0 and 1 , so that $p(t)$ is increasing on this interval.

With $p(t)$ increasing on $[0,1]$, the firm may learn and potentially disclose its information at any time during this interval. Given the firm's equilibrium disclosure strategy, absent disclosure the firm's market value will be its expected value given that it has not yet disclosed, which we denote by $v(t)$. Then a firm with signal $S$ will benefit from disclosing at date $\tau$ rather than wait until after the news announcement only if

$$
\int_{\tau}^{1} \lambda(t)[u(S)-u(v(t))] d t>E\left[\int_{1}^{\infty} \lambda(t)\left[u\left(x^{*}(Y, t)\right)-u(S)\right]^{+} d t \mid S\right]
$$

Now, while the right-hand side of (27) is strictly positive and independent of $\tau$, the lefthand side of (27) tends to 0 as $\tau$ approaches 1 . Therefore, for any signal, there is a point in time such that the firm would rather wait for the release of the public news before deciding whether to disclose its information. Intuitively, the option value of waiting exceeds the benefit of increasing its stock price for a very short interval of time.

This observation implies that the equilibrium threshold strategy, $x^{*}(t)$, prior to the release of public news satisfies $x^{*}(t) \rightarrow \infty$ as $t \rightarrow 1$. We refer to this as an information blackout, 
as it states that voluntary disclosures should be very rare just prior to public news announcements whose timing is known. ${ }^{14}$

A second key implication is that in this setting, because $x^{*}(Y, 1)$ is finite, there will be a positive probability of an immediate disclosure after the public news is released, and the probability of an immediate disclosure is higher for lower realizations of $Y$. This strengthens the result in Section 4, where an immediate disclosure only occurs for $Y$ sufficiently low.

\subsection{Stochastic News Arrival}

Until now we assumed the arrival date of the public news is common knowledge. This is often the case, for example, for government news releases and other forms of aggregate data. A natural question is how our results would change if the timing of the public news is random.

Suppose, for example, the arrival date of the public news has an exponential distribution with arrival rate $\gamma$, so that the probability that the news will arrive in the interval between $t$ and $t+d t$, given that it has not yet arrived, is $\gamma d t$.

Consider a firm's decision whether to preempt the external information and release its information at time $t$ as compared to the alternative of waiting until $t+d t$. The gain from preempting at time $t$ is given by

$$
\lambda(t)[u(S)-u(v(t))] d t
$$

The potential loss is that the public news is released between $t$ and $t+d t$ and is sufficiently positive that the firm regrets having disclosed its signal. The expected loss is given by:

$$
\gamma d t E\left[\int_{t+d t}^{\infty} \lambda(t)\left[u\left(x^{*}(Y, t)\right)-u(S)\right]^{+} d t \mid S\right]
$$

The equilibrium disclosure threshold should therefore satisfy

\footnotetext{
${ }^{14}$ We note that in this case the equilibrium disclosure threshold is likely to be non-monotonic on [0,1]. For $t$ near 0 , the disclosure threshold may fall as $p(t)$ increases, and then rise as $t$ approaches 1 and the option value of delaying dominates.
} 


$$
x^{*}(t)=u^{-1}\left(u(v(t))+\gamma \lambda(t)^{-1} E\left[\int_{t}^{\infty} \lambda(\tau)\left[u\left(x^{*}(Y, t)\right)-u(S)\right]^{+} d \tau \mid S=x^{*}(t)\right]\right)
$$

This equation is nearly identical to the equation we obtained in (18). Hence, the equilibrium behavior is essentially the same as in the case we examined. If, for example, the weights $\lambda$ correspond to standard exponential discounting, then the only change over time comes from the increase in $p(t)$. In that case, the disclosure threshold $x^{*}(t)$ would gradually decline as $p(t)$ increased prior to the public news release.

\subsection{Multiple firms}

A natural extension is a model with no external signal but with multiple firms. For simplicity suppose there are two symmetric firms: A and B whose signals are given by $S^{A}$ and $S^{B}$. As we shall see the equilibrium is similar to the equilibrium of stochastic news arrival that we have just examined. However, the construction of the equilibrium presents a significant computational challenge.

The key observation is the fact that from firms A's perspective, B's signal is an external signal. Let $\gamma^{\mathrm{B}}\left(t \mid S^{\mathrm{B}}\right)$ denote the equilibrium arrival density of B's disclosure conditional on B's signal, so that the probability that B will disclose in the interval between $t$ and $t+$ $d t$, given that it has not yet disclosed, is $\gamma^{\mathrm{B}}\left(t \mid S^{\mathrm{B}}\right) d t$.

Consider firm A's decision whether to preempt and release its information at time $t$ as compared to the alternative of waiting until $t+d t$. A's decision to disclose is similar the case of stochastic arrival of an external signal:

$$
x^{*}(t)=u^{-1}\left(u(v(t))+\lambda(t)^{-1} E\left[\gamma^{B}\left(t \mid S^{B}\right) \int_{t}^{\infty} \lambda(\tau)\left[u\left(x^{*}\left(S^{B}, t\right)\right)-u(S)\right]^{+} d \tau \mid S^{A}=x^{*}(t)\right]\right)
$$

Once the threshold $x^{*}(t)$ is determined, it will then determine the arrival density of A's announcement, $\gamma^{\mathrm{A}}\left(t \mid S^{\mathrm{A}}\right)$. A symmetric equilibrium then requires the solution of the additional fixed point problem $\left(\gamma^{A}=\gamma^{B}\right)$, which is computationally quite challenging. In such a setting, our qualitative results regarding immediate disclosure would continue to apply and would lead to the clustering of news announcements by firms, with clustering more likely the more negative the news. 


\subsection{Alternative Payoffs and Relative Performance}

We have assumed so far that the manager's payoff depends only upon the expected value of the firm conditional on the manager's information. To see why this might be the case, suppose the manager is compensated based on some increasing function of his perceived ability $\alpha$, and let $S=\alpha+\eta$, where $\alpha$ and $\eta$ are joint normal. Suppose also that $Y=S+\theta$, where the noise $\theta$ is independent of $(\alpha, \eta)$. Then the signal $S$ is a sufficient statistic for the manager's ability, and $E[\alpha \mid S, Y]$, is a linear function of $S$. Thus, the manager's objective of maximizing utility over his expected compensation is equivalent to maximizing an increasing function of the market value of the firm.

In the previous example the manager's ability affects the firm's absolute performance. A natural alternative to consider is one in which the manager's ability determines the firm's relative performance. We argue that the qualitative conclusions of our model will continue to apply. To see why, consider for example the extreme case in which $S=Y+$ $\alpha$, where $Y$ and $\alpha$ are independent and joint normal.

Suppose first that $Y$ is revealed at $t=0$ and there is no possibility of preemption. Because $\alpha=S-Y$, we can reinterpret the manager's signal as $\alpha$ and it is immediate that the disclosure threshold $\alpha *(t)$ and therefore the disclosure rate will not depend on $Y$. Thus, our results in Section 3 carry through as before.

Now suppose that $Y$ is realized at $t=1$ so that $S$ can be disclosed before $Y$ is revealed. Then the equilibrium will be similar to our analysis in Section 4 . The disclosure threshold $x^{*}(0)$ at date 0 will reflect a real option premium (i.e., $x^{*}(0)>v(0)$ ), as the agent with type $S=v(0)$ would regret disclosing if $Y$ is sufficiently high (specifically, if $S-Y<$ $\left.\alpha^{*}(1)\right)$. As in our current model, at date $t=1$ there will be a positive probability of immediate disclosure if the market news $Y$ is sufficiently low (so that $Y+\alpha^{*}(1)<x^{*}(0)$ ). On the other hand, if $Y$ is high, disclosure will be delayed. Thus, all of the qualitative conclusions of our model continue to hold if the agent is compensated based on relative rather than absolute performance. 


\section{Implications for Asset Pricing}

An important implication of our results is that with strategic timing of disclosures by managers, the process of information arrival to markets is different from the process of information arrival to firms and managers. For instance, the underlying information process may have constant variability over time and no skewness, but this need not be true of the process describing disclosed information. Below, we discuss the specific implications of our dynamic disclosure model for the skewness and volatility of observed stock returns.

Return Skewness. The basic model of strategic delay developed in Section 2 of the paper implies that individual stock returns will tend to exhibit positive skewness, as firms release good news but delay the disclosure of bad news. The average positive skewness in individual stock returns was documented early by Beedles (1979) and is reproduced for more recent data in Figure 4. Such positive skewness should disappear in our model at the point that $p(t)=1$, and full disclosure occurs. This pattern is consistent with McNichols (1988), who finds less positive skewness in earnings announcement periods (when disclosures are likely to be involuntary) compared to non-announcement periods (when disclosures are more likely to be strategic).

While this effect of disclosure timing on average positive skewness of individual stock returns has been suggested elsewhere (for example, in Damodaran, 1985), our model with public news in Section 4 implies an important, additional conditional pattern. In periods without public news, stock returns will be positively skewed as the firm voluntarily releases good news. When public news is announced, however, returns will be negatively skewed. The reason is that when the public news is good, it is more likely that the firm would have preemptively released good news, mitigating the effect of the news on the stock price. When the public news is bad, however, the firm is less likely to have previously disclosed its information, in which case the stock's return will respond to the public news fully.

Conditional Correlation (Beta). This asymmetry in the response of disclosures to the nature of public news implies that individual stock returns will be more sensitive to aggregate market news when the market news is negative. This implication also finds 
empirical support. Ang and Chen (2002) document that correlations between U.S. stocks and the aggregate U.S. market are much greater for downside moves, especially for extreme downside moves, than for upside moves, and that these correlations differ from the conditional correlations implied by a normal distribution. Interestingly, they find that the downside correlation is stronger for small stocks, where managerial ability to strategically time disclosures may be greater due to investor inattention, and for past loser stocks, where there may be greater adverse information that is being delayed for release until market news arrives.

Further, the asymmetry in response to public news and the resulting downside correlation of firm returns helps explain the empirical result that while individual stock returns tend to be positively skewed on average, stock market indices tend to have negatively skewed returns (Alles and Kling, 1994, and also see Figure 4 for recent evidence regarding index returns). The existing literature has found it hard to reconcile the differential nature of skewness in firm-level and market-level stock returns, and in fact, often interpreted the difference as lack of consensus on evidence of skewness. In contrast, this differential pattern of skewness in returns arises naturally in our model.

Volatility and the Leverage Effect. Finally, our result regarding the acceleration of disclosure after bad market news implies that return volatility will increase after negative shocks. This is consistent with the so-called "leverage effect" (Black, 1976) that conditional on negative returns, return volatility tends to increase. In most striking evidence of this effect, Officer $(1973)$ and Schwert $(1989,1990)$ document that stock market's return variability has been unusually high during downturns such as the 1929-33 Great Depression and the stock market crash of 1987. They contend that the amplitude of the fluctuations in aggregate stock volatility is difficult to explain using simple models of stock valuation, especially during downturns. Our model provides a potential explanation for these findings since the arrival of adverse public news during market downturns should accelerate the disclosure of information by firms and result in greater volatility. Finally, the feature that stock return volatility is stochastic and negatively correlated with the level of returns, is now considered essential in explaining observed option prices. For example, Heston (1993) 


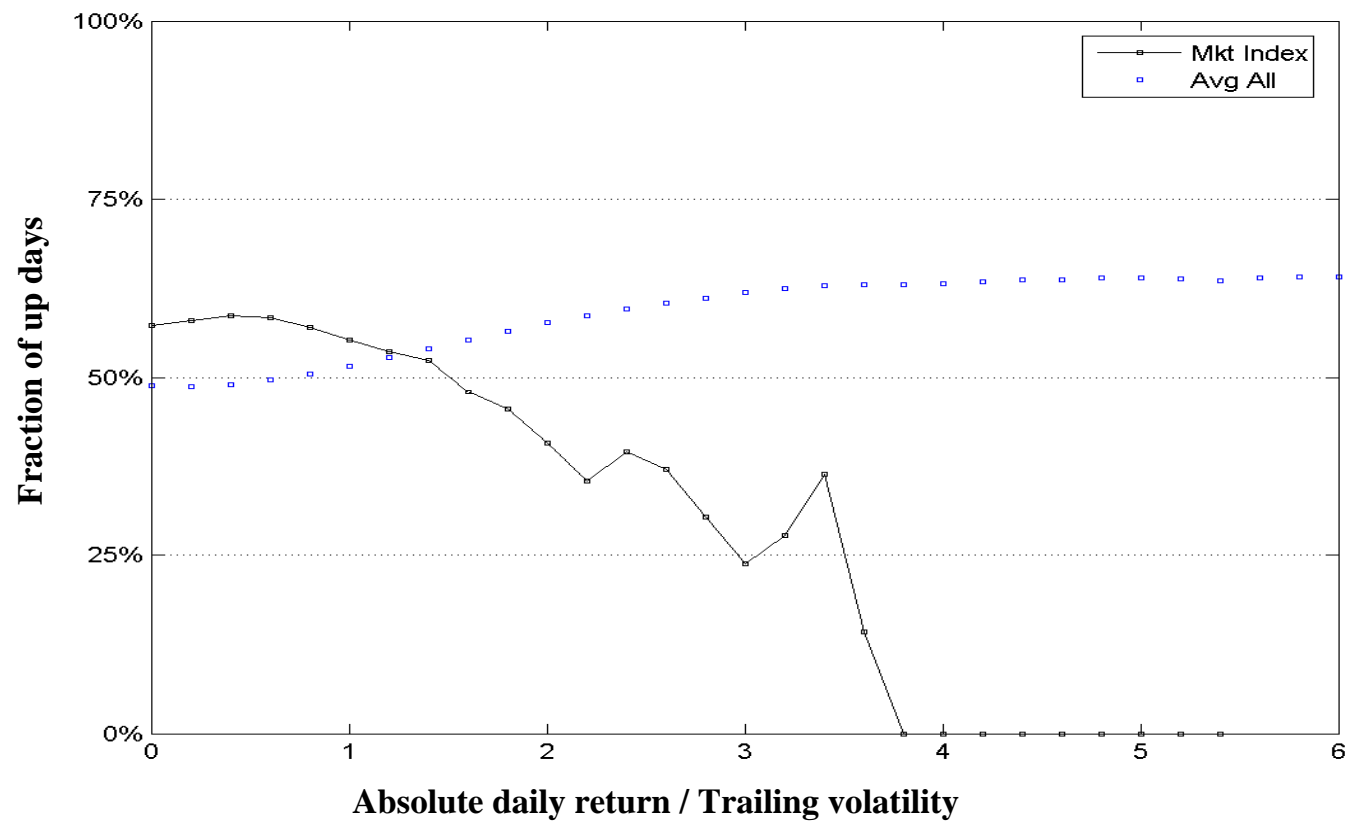

Figure 4: Positive skewness of individual stock returns and negative skewness of index returns

The figure shows the fraction of up (positive stock return) days as a function of absolute stock return divided by the trailing volatility of stock returns (computed as the standard deviation of returns over the prior 100 days). The dotted line shows the fraction of up days for each stock on the New York Stock Exchange over the period 1998-2007 and averaged across all stocks. The solid line plots the fraction calculated for a value-weighted index of stock returns over the same period. Note that the majority of large moves for individual stocks tend to be positive, whereas large moves in the index tend to be negative.

shows that a stochastic volatility model where shocks to volatility are negatively correlated to shocks to returns can fit index option prices well in that it can explain the (Black-Scholes model-based) implied volatility "skew" in index option prices. ${ }^{15}$ Our model's implications thus carry over to options markets as well.

To summarize, skewness and volatility related patterns observed in stock returns are consistent with the dynamics of disclosures by firms and the incentives of managers who have discretion over disclosure timing. In contrast to the existing literature which has

\footnotetext{
${ }^{15}$ Implied volatility "skew" in index option prices is used to describe the pattern that volatility numbers to be put into the Black-Scholes model to fit observed index option prices exhibit a declining relationship with the option strike price. This is now universally considered to be a violation of the Black-Scholes assumption that stock return volatility is constant over time or is deterministic.
} 
often treated such patterns as a statistical artifact of data, our model provides a common information-theoretic foundation for their existence. ${ }^{16}$ Further work is warranted in order to separate this mechanism for volatility and skewness related stock-return patterns from the ones related to trading frictions suggested elsewhere in the literature.

\section{Conclusion}

In this paper, we provided a dynamic disclosure model in which the announcement of bad news hastens the disclosure of information by firms, resulting in bunching of disclosures. Since positive correlation of public news and firms' private information is a critical factor driving this result, our model implies that disclosures should be more clustered within industries and geographies, as empirically found by Tse and Tucker (2007) and Kedia and Rajgopal (2007), respectively.

We assumed throughout our analysis that delaying disclosure is costless for firms. In practice, non-disclosure might entail real costs since the firm would have to bypass observable activities warranted by this information, for example, continue to make investment even in response to adverse information about its prospects. ${ }^{17}$ Another possibility is that there may be litigation risk associated with delay in releasing information. Some researchers (Skinner, 1994, Trueman, 1997) have argued that litigation risk can explain why firms voluntarily disclose bad news. ${ }^{18}$ Conversely, there might also be strategic benefits to a firm from not disclosing information when such information has not yet reached its competitors or the market as a whole. Dierker (2002) analyzes a dynamic disclosure model with such considerations. Modeling more explicitly

\footnotetext{
${ }^{16}$ Shin $(2003,2006)$, discussed in the related literature section, represent contributions that share this theme. Both papers consider single-firm (one-time) disclosure models with verifiable reports where a manager attempts to maximize the current share price and the markets rationally anticipate manager's disclosure policy. The models generate implications such as the appearance of short-run momentum and long-run reversal in returns and the higher return variance following a poor disclosed outcome. Rogers, Schrand and Verrecchia (2007), cited above, find evidence for some of the implications of Shin's models for firm-level and market-level return and return volatility.

${ }^{17}$ Rajan (1994) examines coordination and strategic delay in the recognition of bad loans by banks. He assumes that in order to hide bad loans, banks must continue lending or make new loans to the defaulted borrowers, which is costly in real terms. He shows that when bank loan portfolios are correlated, strategic delay in recognition of bad loans can induce a low-frequency cycle in the real sector, characterized by excessive extension of credit in good times.

${ }^{18}$ Such effects are captured to some extent in a reduced-form fashion in our model by the parameter $q$, which represents the likelihood with which the firm has no discretion over the release of its information.
} 
the cost or benefits of non-disclosure within our framework could potentially lead to further empirical predictions. We leave such an extension for future work.

An alternative motive for delayed disclosure that has been proposed is managerial shorttermism. While our model of the manager's objective is general enough to include such features, it is interesting to note that in our setting the impact of short-termism is ambiguous. For example, decreasing the weight $\lambda(t)$ that the manager puts on the stock price after the public announcement $(t>1)$ will reduce the real option effect of delay and lead to greater disclosure in period 0 .

Finally, we believe our dynamic disclosure game has interesting asset-pricing implications for skewness and volatility of firm-level and market-wide stock returns. Fully establishing the empirical link between strategic timing of disclosures and these features of stock returns appears to be a promising line of enquiry for further work. 


\section{Appendix}

Proof of Proposition 1: First note that $h_{S}(x, \rho)<E[S]$ for $x$ in the interior of the support of $S$, and that $h_{S}(x, \rho) \rightarrow E[S]$ as $x \rightarrow \pm \infty$. Therefore $h_{S}$ has a minimum in the interior of the support of $S$. Suppose that $x^{*}$ satisfies $h_{S}\left(x^{*}, \rho\right)=x^{*}$; then $x^{*}$ represents the average type in a pool of types who were either uninformed, or privately informed with a signal lower than $x^{*}$.

Now consider $h_{S}(x, \rho)$, with $x>x^{*}$. In this case, relative to the pool of types corresponding to $h_{S}\left(x^{*}, \rho\right)$, we have to include new types in the pool that are informed and exceed $x^{*}$. This must raise the quality of average type above $x^{*}$. Thus, $h_{S}(x, \rho)>$ $h_{S}\left(x^{*}, \rho\right)$. Next consider $h_{S}(x, \rho)$, with $x<x^{*}$. In this case, relative to the pool of types corresponding to $h_{S}\left(x^{*}, \rho\right)$, we have to exclude types from the pool that are informed but below $x^{*}$. Since we are eliminating some types that are lower than the average quality of pool, this must also raise the quality of average type above $x^{*}$. Thus again, $h_{S}(x, \rho)>$ $h_{S}\left(x^{*}, \rho\right)$. Therefore, the equilibrium threshold $x^{*}=h_{S}\left(x^{*}, \rho\right)$ occurs at the unique minimum of the function $h_{S}$.

To see that threshold strategies lead to the worst possible beliefs, consider any disclosure strategy $\Gamma$ (threshold or not) and let $v=E[S \mid$ nondisclosure given $\Gamma]$. Then given these beliefs, the optimal policy $\Gamma^{\prime}$ for the manager is to disclose if $S>v$ and not disclose if $S \leq$ $v$. Unless the two policies coincide almost surely, then under $\Gamma^{\prime}$ some above average types are removed from the pool of non-disclosers, and some below average types are added, so that $E\left[S \mid\right.$ nondisclosure given $\left.\Gamma^{\prime}\right]<v$.

Finally, because $h_{S}$ decreases with $\rho$, so does the threshold. Iqed

Proof of Proposition 2: Because the payoff from non-disclosure does not depend on the firm's true type, it is immediate that the optimal strategy can be expressed as a threshold. Because $p(t)$ is increasing, the optimal threshold in (7) is decreasing with $t$. As a result, only the current threshold is relevant in determining the firm's market value 
absent disclosure, which is given by $v_{t}=h_{S}\left(x^{*}(t), \rho(t)\right)$. Finally, because $v(t)$ also declines with $t$, it is optimal for the firm to disclose if and only if $S$ exceeds $v(t)$, and so $x^{*}(t)$ is indeed an equilibrium threshold.

To see that the equilibrium is unique, note that by the same reasoning as in the static case, any equilibrium must involve a threshold strategy (the gain from disclosure is increasing in type). Let $x(t)$ be some other disclosure policy, and let $v(t)$ be the market value of the firm in the event of non-disclosure under this policy. Note first that if $x(t)$ is an equilibrium, and if $t^{\prime}>t$, then

$$
x(t) \geq v(t) \geq X *(t) \geq X *\left(t^{\prime}\right) .
$$

The first inequality follows because the manager would not disclose if it would lower the current share price. The second follows because, from Proposition 1, the share price $X^{*}(t)$ is the lowest possible share price under any beliefs regarding the manager's disclosure policy. Finally, the last follows because $x *(t)$ is weakly decreasing.

Next we claim that

$$
x(t)=v(t) \text { if and only if } x(t)=x^{*}(t) .
$$

To see why, note with this disclosure threshold at date $t$, because $x(t)=x^{*}(t)<x(s)$ for all $s<t$ from (28), the set of non-disclosing firms is precisely the same as the set that is privately informed with $S<x^{*}(t)$, and thus $x^{*}(t)=h\left(x^{*}(t), \rho(t)\right)=v(t)$. The "only if" follows because, as in the static case, this fixed point is unique (lowering the threshold from any fixed point must raise the share price).

Finally, note that if $x(t)>v(t)$, it must be that $v\left(t^{*}\right)=\sup \left\{v\left(t^{\prime}\right): t^{\prime}>t\right\}>x(t)$ (it pays to delay disclosure only if a higher price can be obtained in the future from not disclosing). But then because $x(t *)=v(t *)$ (there is no reason to delay at $t *$ ), we have from (28) and (29) that $v(t *)=x^{*}(t *) \leq x(t)$, a contradiction.

Iqed 
Proof of Proposition 4: For a fixed $t \geq 1$, let $\rho_{0}=p(0) q /(1-p(t)), \quad \rho_{t}=$ $(p(t)-p(0)) q /(1-p(t))$, and with some abuse of notation we define

$$
h\left(x, x_{0}, Y\right) \equiv \frac{E[S \mid Y]+\rho_{0} \operatorname{Pr}\left(S \leq x_{0} \mid Y\right) E\left[S \mid S \leq x_{0}, Y\right]+\rho_{t} \operatorname{Pr}(S \leq x \mid Y) E[S \mid S \leq x, Y]}{1+\rho_{0} \operatorname{Pr}\left(S \leq x_{0} \mid Y\right)+\rho_{t} \operatorname{Pr}(S \leq x \mid Y)}
$$

Then $h\left(x, x_{0}, Y\right)$ is the expected value of a firm given news $Y$, if it learned its signal was below $x_{0}$ at date 0 , or it learned its signal was below $x$ after date 0 but by date $t$. Because a firm will disclose if its signal is above the lowest threshold that occurs after it learns its information, firms that learn their information at date 0 will disclose if $S>\min \left(x^{*}(0)\right.$, $\left.x^{*}(Y, t)\right)$, and those that learn their information later will disclose if $S>x^{*}(Y, t)$. So, letting $x_{0}^{*}=x^{*}(0)$ to shorten notation, we have ${ }^{19}$

$$
h\left(x^{*}(Y, t), x^{*}(Y, t) \wedge x_{0}^{*}, Y\right)=E[S \mid Y, \text { nondisclosure by date } t]
$$

Using the intuition from Proposition 1, define the function $h *$ to be the most pessimistic assessment of the disclosure threshold

$$
h^{*}\left(x_{0}^{*}, y\right) \equiv \min _{\tilde{x}} h\left(\tilde{x}, \tilde{x} \wedge x_{0}^{*}, y\right)
$$

Then, by an identical argument to Proposition 1, the equilibrium post-news disclosure threshold satisfies

$$
x^{*}(y, t)=h^{*}\left(x_{0}^{*}, y\right)=h\left(x^{*}(Y, t), x^{*}(Y, t) \wedge x_{0}^{*}, Y\right)
$$

Now, using Proposition 3,

$$
h^{*}\left(x_{0}^{*}, y\right) \geq \min _{\tilde{x}} h(\tilde{x}, \tilde{x}, y)=a+\beta y+\sigma z^{*}(t)
$$

and the inequality is strict if and only if $x_{0}^{*}<a+\beta y+\sigma z^{*}(t)$, or equivalently $y>y_{0}(t)$. Therefore,

$$
x^{*}(y, t)=h^{*}\left(x_{0}^{*}, y\right)=a+\beta y+\sigma z^{*}(t) \text { for all } y \leq y_{0}(t)
$$

For $y>y_{0}(t)$, note that we can write

\footnotetext{
${ }^{19}$ We use the notation $x \wedge y=\min (x, y)$.
} 


$$
h^{*}\left(x_{0}^{*}, y\right)=h^{*}\left(x_{0}^{*}, y_{0}\right)+\int_{y_{0}}^{y}\left(\frac{\partial}{\partial \tilde{y}} h^{*}\left(x_{0}^{*}, \tilde{y}\right)\right) d \tilde{y}
$$

Now, (9) implies that $h^{*}\left(x_{0}+\beta \delta, y+\delta\right)=h^{*}\left(x_{0}, y\right)+\beta \delta$. Therefore,

$$
\beta\left(\frac{\partial}{\partial x_{0}} h^{*}\left(x_{0}, y\right)\right)+\left(\frac{\partial}{\partial y} h^{*}\left(x_{0}, y\right)\right)=\beta
$$

Combining (34)-(36) we have

$$
\begin{aligned}
h^{*}\left(x_{0}^{*}, y\right) & =h^{*}\left(x_{0}^{*}, y_{0}\right)+\int_{y_{0}}^{y}\left[\beta-\beta\left(\frac{\partial}{\partial x_{0}} h^{*}\left(x_{0}^{*}, y\right)\right)\right] d \tilde{y} \\
& =a+\beta y+\sigma z^{*}(t)-\beta \int_{y_{0}}^{y}\left(\frac{\partial}{\partial x_{0}} h^{*}\left(x_{0}^{*}, y\right)\right) d \tilde{y}
\end{aligned}
$$

Therefore, we have

$$
k(y, t) \equiv-\beta \int_{y_{0}}^{y}\left(\frac{\partial}{\partial x_{0}} h^{*}\left(x_{0}^{*}, y\right)\right) d \tilde{y}
$$

Note further that for $y>y_{0}(t)$, because

$$
x_{0}^{*}<a+\beta y+\sigma z^{*}(t)=\min _{\tilde{x}} h(\tilde{x}, \tilde{x}, y)<h^{*}\left(x_{0}^{*}, y\right),
$$

increasing $x_{0}^{*}$ lowers the average quality of the non-disclosing firms and hence $\frac{\partial}{\partial x_{0}} h^{*}\left(x_{0}, y\right)<0$. Thus, $k$ is strictly increasing in $y$, as claimed. Finally, we verify that the threshold is decreasing in $p(t)$ by noting that $h\left(\tilde{x}, \tilde{x} \wedge x_{0}^{*}, y\right)$ declines with $p(t)$. Iqed

Proof that a threshold strategy is optimal prior to news announcement (Section 4.3):

Here we provide a justification for a threshold strategy prior to the public news announcement. To simplify the analysis, here we assume that the manager's payoff function $u$ is linear. Given this assumption, it is sufficient to analyze the case $u(v)=v$.

Consider the condition (17). We need to show that if this condition holds for some type $\mathrm{S}$, it holds for all higher types. Now, the left hand side of (17) clearly increases with $S$. 
Thus, it is enough to show that the right hand side of (17) weakly decreases with $S$. Without loss of generality normalize $S$ so that $a=0$ and note that we can write $Y=\frac{\beta}{\beta^{2}+\sigma^{2}} S+\eta$ where $S$ and $\eta \sim N\left(0, \frac{\sigma^{2}}{\beta^{2}+\sigma^{2}}\right)$ are independent. Therefore,

$$
\begin{aligned}
x^{*}(Y, t)-S & =\beta Y+\sigma z^{*}(t)+k(Y, t)-S \\
& =\eta+\sigma z^{*}(t)+k\left(\frac{\beta}{\beta^{2}+\sigma^{2}} S+\eta, t\right)-\frac{\sigma^{2}}{\beta^{2}+\sigma^{2}} S
\end{aligned}
$$

and so the result follows if

$$
\frac{\beta}{\beta^{2}+\sigma^{2}} k^{\prime}(Y, t)-\frac{\sigma^{2}}{\beta^{2}+\sigma^{2}} \leq 0 \text { or equivalently } k^{\prime}(Y, t) \leq \frac{\sigma^{2}}{\beta}
$$

This condition obviously holds if $Y \leq y_{0}(t)$, since then $k^{\prime}=0$. Consider the case $Y=y>$ $y_{0}(t)$ and therefore $x^{*}(y, t)>x_{0}^{*}$. Using the expression for $k$ in (38), we can write (40) as

$$
\frac{\partial}{\partial x_{0}} h^{*}\left(x_{0}^{*}, y\right) \geq-\frac{\sigma^{2}}{\beta^{2}}
$$

Now, from (31),

$$
\frac{\partial}{\partial x_{0}} h^{*}\left(x_{0}^{*}, y\right)=\frac{\partial}{\partial x_{0}} h\left(x^{*}(y, t), x_{0}^{*}, y\right)=\frac{\rho_{0} n\left(\frac{x_{0}^{*}-\beta y}{\sigma}\right)\left(\frac{x_{0}^{*}-h\left(x^{*}(y, t), x_{0}^{*}, y\right)}{\sigma}\right)}{1+\rho_{0} N\left(\frac{x_{0}^{*}-\beta y}{\sigma}\right)+\rho_{t} N\left(\frac{x^{*}(y, t)-\beta y}{\sigma}\right)}
$$

Because $x_{0}^{*}<h\left(x^{*}(y, t), x_{0}^{*}, y\right)=x^{*}(y, t)$, and because $h$ decreases with $\rho_{t}$, the magnitude of (42) decreases with $\rho_{t}$. Thus, it is sufficient to establish (41) for the case $\rho_{t}=0$. Also, because with $\rho_{t}=0, h\left(x^{*}(y, t), x_{0}^{*}, y\right)=\beta y+\sigma h_{\varepsilon}\left(\frac{x_{0}^{*}-\beta y}{\sigma}, \rho_{0}\right)$, we can substitute $z=\frac{x_{0}^{*}-\beta y}{\sigma}$ in (42) and so a sufficient condition for (42) is

$$
\frac{\rho_{0} n(z)\left(z-h_{\varepsilon}\left(z, \rho_{0}\right)\right)}{1+\rho_{0} N(z)}=\frac{\partial}{\partial z} h_{\varepsilon}\left(z, \rho_{0}\right) \geq-\frac{\sigma^{2}}{\beta^{2}} \text { for all } z .
$$


Because $h_{\varepsilon}<0$,

$$
\frac{\rho_{0} n(z)\left(z-h_{\varepsilon}\left(z, \rho_{0}\right)\right)}{1+\rho_{0} N(z)}>-\rho_{0}|n(z) z| \geq-\rho_{0} n(1)>-\frac{\rho_{0}}{4}
$$

a very weak sufficient condition for a threshold strategy to be optimal is that $\sigma>\frac{1}{2} \beta \sqrt{\rho_{0}}$. This condition is extremely weak, in part because we have required monotonicity of the right hand side of (17) state-by-state, rather than in expectation. Indeed, in the extreme alternative case $\sigma=0$, it is easy to see that a threshold strategy is optimal: $Y$ is then a perfect signal of $S$, and the option value of waiting disappears. Iqed 


\section{References}

Admati, Anat and Paul Pfleiderer, "Forcing firms to talk: financial disclosure regulation and externalities," Review of Financial Studies, 13, 479-519, 2000.

Aboody, David and Ron Kasznik, "CEO Stock Option Awards and the Timing of Corporate Voluntary Disclosures," Journal of Accounting and Economics, 29, 73$100,2000$.

Alles, L. A. \& Kling, J. L.. "Regularities in the Variation of Skewness in Asset Returns," Journal of Financial Research, 17, 427-438, 1994.

TAng, Andrew and Joseph Chen, "Asymmetric Correlations of Equity Portfolios," Journal of Financial Economics, 63, 3, 443-494, 2002.

Beedles, W. L., "Return, Dispersion and Skewness: Synthesis and Investment Strategy," Journal of Financial Research, 2, 71-80, 1979.

Black, F., "Studies in Stock Price Volatility Changes," Proceedings of the Business and Economic Statistics section of the American Statistical Association, 177-181, 1976.

Burgsthaler, David and Ilia, Dichev, "Earnings management to avoid earnings decreases and losses," Journal of Accounting and Economics, 24, 99-126, 1997.

Damodaran, A., "Economic Events, Information Structure, and the Return-generating Process," The Journal of Financial and Quantitative Analysis, 20(4), 423-434, 1985.

Diamond, D. W, "Optimal Release of Information by Firms," Journal of Finance, 40, 1071-1094, 1985.

Dierker, Martin, "Dynamic Information Disclosure," Working paper, University of California at Los Angeles, 2002.

Dye, R., "Mandatory Versus Voluntary Disclosures: The Cases of Financial and Real Externalities," Accounting Review, 1-24, (1990).

Dye, R. and S. Sridhar, "Industry-wide Disclosure Dynamics," Journal of Accounting Research, 157-74, Spring 1995. 
Genotte, B. and B. Trueman, "The Strategic Timing of Corporate Disclosures," Review of Financial Studies, 9(2), 665-90, 1996.

Grossman, S. J., "The Informational Role of Warranties and Private Disclosures about Product Quality," Journal of Law and Economics, 24, 461- 483, 1981.

Heston, S.L., "A closed-form solution for options with stochastic volatiltiy and applications to bond and currency options," Review of Financial Studies, 6, 327-343, 1993.

Kedia, Simi and Shiva Rajgopal, "Geography and the Incidence of Financial Misreporting,” Working Paper, Rutgers Business School, 2007.

McNichols, Maureen, "A comparison of the skewness of stock return distributions at earnings and non-earnings announcement dates," Journal of Accounting and Economics, 10, 239-273, 1988.

Milgrom, P. R., "Good News and Bad News: Representation Theorems and Applications," Bell Journal of Economics, 12, 350-391, 1981.

Miller, Gregory S., "Earnings Performance and Discretionary Disclosure," Journal of Accounting Research,, 40, 173-204, 2002.

Officer, R. R., "The variability of the market factor of the New York stock exchange," Journal of Business, 46, 434-453, 1973.

Rajan, Raghuram G., "Why Bank Credit Policies Fluctuate: A Theory and Some Evidence,” The Quarterly Journal of Economics, 109(2), 399-441, 1994.

Rogers, Jonathan L., Schrand, Catherine M. and Verrecchia, Robert E., "Strategic Disclosure as an Explanation for Asymmetric Return Volatility," Working Paper, University of Chicago - GSB, 2007.

Ross, S. A., "Disclosure Regulation in Financial Markets: Implications of Modern Finance Theory and Signaling Theory," in F. K. Edwards (ed.), Issues in Financial Regulation, McGraw-Hill, New York, 1979.

Schwert, G William, "Why Does Stock Market Volatility Change over Time?," Journal of Finance, 44(5), 1115-53, 1989. 
Schwert, G William, "Stock Volatility and the Crash of '87," Review of Financial Studies, 3(1), 77-102, 1990.

Skinner, Douglas, "Why Firms Voluntarily Disclose Bad News," Journal of Accounting Research, 32(1), 38-60, 1994.

Song-Shin, Hyun, "Disclosures and Asset Returns," Econometrica, 71(1), 105-133, 2003.

Song-Shin, Hyun, "Disclosure Risk and Price Drift", Journal of Accounting Research, 44, 351-379, 2006.

Teoh S. H. and C. Y. Hwang, "Nondisclosure and adverse disclosure as signals of firm value," Review of Financial Studies, 4, 283-313, 1991.

Tse, Senyo and Jennifer Tucker, "Within-Industry Timing of Earnings Warnings: Do Managers Herd?,” Working Paper, Texas A\&M University, 2007.

Trueman, B., "Managerial Disclosures and Shareholder Litigation," Review of Accounting Studies, 2, 181-199, 1997. 